PROCEEDINGS OF THE

AMERICAN MATHEMATICAL SOCIETY

Volume 128, Number 11, Pages 3201-3203

S 0002-9939(00)05437-X

Article electronically published on May 18, 2000

\title{
A CO-FROBENIUS HOPF ALGEBRA WITH A SEPARABLE GALOIS EXTENSION IS FINITE
}

\author{
M. BEATTIE, S. DĂSCĂLESCU, AND Ş. RAIANU
}

(Communicated by Ken Goodearl)

\begin{abstract}
If $H$ is a co-Frobenius Hopf algebra over a field, having a Galois $H$-object $A$ which is separable over $A^{c o H}$, its ring of coinvariants, then $H$ is finite dimensional.
\end{abstract}

Let $H$ be a Hopf algebra over a field $k$ and $(A, \rho)$ a right $H$-comodule algebra such that $A$ is $H$-Galois over its ring of coinvariants $A^{c o H}$. If $H$ is a group algebra $k G$ so that then $A$ is strongly graded, it is well known that if $A$ is separable over $A_{e}$, its ring of coinvariants, then the group $G$ is finite. We show in this note that this result holds for all co-Frobenius Hopf algebras $H$.

Recall that $H$ is co-Frobenius if $H^{* r a t}$, the rational submodule of $H^{*}$ as a (left or right) $H^{*}$-module, is nonzero. If $H^{* r a t}$ is nonzero, it is dense in $H^{*}$. A right $H$ comodule algebra $A$ is right $H$-Galois if the canonical map can: $A \otimes_{A^{c o H}} A \rightarrow A \otimes H$, $a \otimes b \longmapsto \sum a b_{0} \otimes b_{1}$, is a bijection. Any right $H$-comodule $M$ is a left $H^{*}$-module via $p \cdot m=\sum m_{0}\left\langle p, m_{1}\right\rangle$ for $p \in H^{*}, m \in M$. Also $H^{*}$ and $H^{* r a t}$ are right $H$ modules via $(p<h)(f)=\langle p, h f\rangle$ for $p \in H^{*}$ or $H^{* r a t}$ and $h, f \in H$. Details about $H$-Galois objects with $H$ co-Frobenius can be found in [1].

Lemma 1. Suppose $H$ is a Hopf algebra such that $H=\bigoplus_{\lambda \in I} H_{\lambda}$ where $H_{\lambda}$ is a left $H$-subcomodule. Then if $A$ is a right $H$-Galois object, $A=\bigoplus_{\lambda \in I} A_{\lambda}$ where $A_{\lambda}=\left\{a \mid a \in A, \rho(a) \in A \otimes H_{\lambda}\right\}$ and $A_{\lambda} \neq 0$ for all $\lambda \in I$.

Proof. Let $p_{\lambda} \in H^{*}$ be the projection defined by $p_{\lambda}(h)=0$ if $h \in H_{\mu}, \mu \neq \lambda$, and $p_{\lambda}(h)=\epsilon(h)$ for $h \in H_{\lambda}$. Then $p_{\lambda} \cdot H=\left\{\sum h_{1} p_{\lambda}\left(h_{2}\right) \mid h \in H\right\}=H_{\lambda}$ since if $h \in H_{\mu}, \mu \neq \lambda, p_{\lambda} \cdot h=0$, but if $h \in H_{\lambda}, p_{\lambda} \cdot h=\sum h_{1} \epsilon\left(h_{2}\right)=h$.

Now, $p_{\lambda} \cdot A=\left\{\sum a_{0} p_{\lambda}\left(a_{1}\right) \mid a \in A\right\}$ and so for $a \in A$,

$$
\rho\left(p_{\lambda} \cdot a\right)=\sum a_{0} \otimes a_{1} p_{\lambda}\left(a_{2}\right)=\sum a_{0} \otimes p_{\lambda} \cdot a_{1} \in A \otimes H_{\lambda}
$$

so that $p_{\lambda} \cdot A \subseteq A_{\lambda}$. Clearly if $a \in A_{\lambda}, p_{\lambda} \cdot a=a$ so $A_{\lambda}=p_{\lambda} \cdot A$, and $A=\bigoplus_{\lambda \in I} A_{\lambda}$.

Since $A$ is Galois, the canonical map can from $A \otimes_{A^{c o H}} A$ to $A \otimes H, a \otimes b \longmapsto$ $\sum a b_{0} \otimes b_{1}$ is a bijection and thus $A_{\lambda} \neq 0$ for all $\lambda \in I$.

Received by the editors August 12, 1998 and, in revised form, January 15, 1999.

1991 Mathematics Subject Classification. Primary 16W30.

The first author's research was partially supported by NSERC.

The last two authors thank Mount Allison University for their kind hospitality. 
If $H$ is co-Frobenius, then $H$ has a direct sum decomposition of left subcomodules

$$
H=\bigoplus_{\lambda \in I} E\left(M_{\lambda}\right)=\bigoplus_{\lambda \in I} H_{\lambda}
$$

where the $M_{\lambda}$ 's are the simple left subcomodules of $H$ and the $E\left(M_{\lambda}\right)$ 's their injective envelopes. By [4, Theorem 3], the $E\left(M_{\lambda}\right)$ are finite dimensional $k$-spaces. Thus $H$ is finite dimensional if and only if $I$ is finite.

Also since $H$ is co-Frobenius, then $H$ is semiperfect as a coalgebra, and the results of [2, Theorem 2.4] apply. In particular, for $H_{\lambda}=E\left(M_{\lambda}\right)$, the maps $p_{\lambda} \in H^{*}$ defined in Lemma 1 lie in $H^{* r a t}$ and the family of finite sums of the $p_{\lambda}$ is a set of local units for $H^{* r a t}$.

Theorem 2. If $H$ is co-Frobenius and $A$ is $H$-Galois and separable over $A^{c o H}$, then $H$ is finite dimensional.

Proof. Suppose that $I$ is infinite. Let $\sum a_{i} \otimes b_{i} \in A \otimes_{A^{\text {coH }}} A$ be the separability idempotent for the extension $A / A^{c o H}$; then

$$
\sum a_{i} b_{i}=1 \quad \text { and } \quad \sum c a_{i} \otimes b_{i}=\sum a_{i} \otimes b_{i} c \text { for all } c \in A .
$$

Since the maps $p_{\lambda}$ can be used to build a set of local units $\sum_{j=1}^{n} p_{\lambda_{j}}$ for $H^{* r a t}$, then if $f \in H^{* r a t}$,

$$
f=\sum_{\lambda \in F} f p_{\lambda}
$$

for some finite subset $F$ of $I$.

Let $W$ be the finite dimensional subspace of $H$ generated by the $b_{i_{1}}$ and $p_{W} \in$ $H^{* r a t}$ a map equal to $\epsilon$ on $W$. The maps $p_{W}<b_{i_{1}}$ are elements of $H^{* r a t}$, and thus there is associated to each a finite subset $F$ of $I$ as above. Let $\lambda_{0}$ be an element of $I$ which does not lie in any of these finite sets. In other words, $p_{W}<b_{i_{1}}$ is zero on $H_{\lambda_{0}}$ for all $b_{i}$.

Let $0 \neq c \in A_{\lambda_{0}}$ and applying $I d \otimes \rho$ to $\sum c a_{i} \otimes b_{i}=\sum a_{i} \otimes b_{i} c$, we obtain

$$
\sum c a_{i} \otimes b_{i_{0}} \otimes b_{i_{1}}=\sum a_{i} \otimes b_{i_{0}} c_{0} \otimes b_{i_{1}} c_{1} .
$$

Now apply $M \cdot\left(I d \otimes I d \otimes p_{W}\right), M$ the multiplication in $H$, to both sides of the above equality and obtain

$$
c=\sum c a_{i} b_{i_{0}} p_{W}\left(b_{i_{1}}\right)=\sum a_{i} b_{i_{0}} c_{0}\left(p_{W}<b_{i_{1}}\right)\left(c_{1}\right)=0,
$$

which is a contradiction. Thus $I$ is finite, and $H$ is finite dimensional.

Corollary 3. If $A / A^{c o H}$ is separable and $H$-Galois, $H$ co-Frobenius, then the equivalent conditions of [3, Proposition 1.8] hold.

\section{REFERENCES}

[1] M. Beattie, S. Dăscălescu and S. Raianu, Galois extensions for co-Frobenius Hopf algebras, J. Algebra 198 (1997), 164-183. MR 99c:16034

[2] M. Beattie, S. Dăscălescu, L. Grünenfelder and C. Năstăsescu, Finiteness conditions, co-Frobenius Hopf algebras and quantum groups, J. Algebra 200 (1998), 312-333. MR 99c:16035 
[3] M. Cohen and D. Fischman, Semisimple extensions and elements of trace 1, J. Algebra 149 (1992), 419-437. MR 93c:16038

[4] B. Lin, Semiperfect coalgebras, J. Alg. 49 (1977), 357-373. MR 58:16749

Department of Mathematics and Computer Science, Mount Allison University, Sackville, New Brunswick, Canada E4L 1E6

E-mail address: mbeattie@mta.ca

University of Bucharest, Faculty of Mathematics, Str. Academiei 14, RO-70109 Bucharest 1, Romania

E-mail address: sdascal@al.math.unibuc.ro

University of Bucharest, Faculty of Mathematics, Str. Academiei 14, RO-70109 Bucharest 1, Romania

E-mail address: sraianu@al.math.unibuc.ro 